\title{
Patterns of maternal depression, anxiety, and stress symptoms from pregnancy to 5 years postpartum in an Australian cohort experiencing adversity
}

\author{
Hannah Bryson ${ }^{1,2} \circledast$ Susan Perlen ${ }^{1,2} \cdot$ Anna Price $^{1,2,3} \cdot$ Fiona Mensah $^{2,3} \cdot$ Lisa Gold $^{4} \cdot$ Penelope Dakin $^{5}$. \\ Sharon Goldfeld ${ }^{1,2,3}$
}

Received: 1 December 2020 / Accepted: 11 May 2021 / Published online: 25 May 2021

(c) The Author(s), under exclusive licence to Springer-Verlag GmbH Austria, part of Springer Nature 2021

\begin{abstract}
The objective of this study is to describe the longitudinal patterns of depression, anxiety, and stress symptoms from pregnancy to 5 years postpartum, in a cohort of Australian mothers experiencing adversity. Longitudinal data were drawn from the control group of a trial of nurse home visiting. Pregnant women experiencing adversity ( $\geq 2$ of 10 adversity risk factors) were recruited from antenatal clinics across 2 Australian states (30 April 2013-29 August 2014). Women completed the Depression Anxiety and Stress Scales short-form (DASS-21) at 11 time-points from pregnancy to 5 years postpartum. DASS-21 scores were summarized at each time-point for all women and by level of adversity risk. Three hundred fifty-nine women $(100 \%)$ completed the DASS-21 in pregnancy and 343 (96\%) provided subsequent data. Mental health symptoms were highest in pregnancy and at 4 and 5 years postpartum. While this pattern was comparable across levels of antenatal adversity risk, women with greatest adversity risk had consistently higher mental health symptoms. In a cohort of mothers experiencing adversity, depression, anxiety, and stress symptoms were highest in pregnancy and at 4 to 5 years postpartum. The striking patterns of persistent, high, mental health symptoms, beyond the first year postpartum, can inform a more equitable and responsive health system.
\end{abstract}

Keywords Maternal mental health $\cdot$ Adversity $\cdot$ Perinatal $\cdot$ Early childhood

\section{Introduction}

Maternal mental health is globally recognized as a public health priority (Ko and Haight 2020; Vigo et al. 2016). Mental health symptoms, such as depression, anxiety, and stress, have well-established impacts on mothers' own wellbeing

Hannah Bryson

hannah.bryson@mcri.edu.au

1 Centre for Community Child Health, The Royal Children's Hospital, Parkville, Vic 3052, Australia

2 Population Health, Murdoch Children's Research Institute, Parkville, Vic 3052, Australia

3 Department of Paediatrics, University of Melbourne, Parkville, Vic 3052, Australia

4 School of Health and Social Development, Deakin University, Burwood, Vic 3125, Australia

5 Australian Research Alliance for Children and Youth, Canberra City, ACT 2601, Australia and their children's health and development. For women themselves, these impacts include poorer quality of life, increased risk of chronic disease and substance abuse, as well as the economic burden of health care costs, loss of earnings, and unemployment (Luca et al. 2019; PricewaterhouseCoopers et al. 2019). For young children, maternal mental health problems can negatively impact mothers' capacity to provide care at a time when children are most sensitive to their environments, resulting in poorer behavioral, cognitive, and emotional development outcomes for children (Kingston and Tough 2014; Stein et al. 2014). Despite the importance of the early years for children's development, there is little research examining the patterns and prevalence of maternal mental health symptoms beyond the first year postpartum. Even further lacking are longitudinal data for women experiencing social and economic adversities (such as unemployment, financial problems, parenting alone; termed "adversity" from here on), who have greater needs for health services but are the least able to access them (Hart 1971; Redshaw and Henderson 2016; The Lancet 
2021). Understanding the patterns of mental health symptoms for women experiencing adversity can inform more precise and effective responses designed to ameliorate the individual, family, and societal harms from poor maternal mental health.

Depression is reported to affect between 10 and $20 \%$ of all mothers in the immediate postpartum period up until 12 months (Shorey et al. 2018; Woody et al. 2017). Perinatal anxiety is also common, both co-morbid with depression and on its own (Austin et al. 2010); the prevalence for any type of anxiety disorder in the early postpartum period has been estimated to be between 4 and 20\% (Austin et al. 2010; Dennis et al. 2017; Leach et al. 2017). The variation in estimates for both depression and anxiety may in part be explained by differences in measures, definitions of mental health, and time-points for data collection (Leach et al. 2017). While stress is also a substantial component of mental health problems, prevalence estimates for maternal stress are less commonly reported; instead stress symptoms are often considered as a component of anxiety under the umbrella term "distress."

Beyond the first 12 months postpartum, the prevalence of maternal mental health problems has been less well studied. However, the available studies suggest that maternal mental health problems persist (Putnick et al. 2020), and that the prevalence may actually increase (Shorey et al. 2018). In a cohort of Australian first-time mothers, rates of depression were highest (15\%) at 4 years compared to any time in the first 12 months postpartum (8-11\%) (Woolhouse et al. 2015). Similarly, in an Australian population-representative cohort, $16 \%$ of mothers experienced persistently high depression symptoms from birth to 7 years postpartum (Giallo et al. 2014); and in a US cohort, $13 \%$ of women experienced increasing or persistently high depression symptoms up to 3 years postpartum (Putnick et al. 2020). A Canadian cohort study further found that $8 \%$ of mothers reported persistently high anxiety symptoms and $13 \%$ persistently high stress symptoms from birth to 3 years postpartum (Mughal et al. 2018).

While these existing studies focus on large, population cohorts, women who are experiencing adversity are often underrepresented, because they are less likely to be included in or remain in research studies (Bonevski et al. 2014). Social and economic adversities commonly co-occur, and multiple adversities are known to have a cumulative detrimental effect on maternal mental health (Kingsbury et al. 2018; Price et al. 2018). The patterns in which mental health symptoms unfold for mothers experiencing adversity remain unclear. To generate this evidence, the current study draws on data from the usual care arm (control group) of the right@ home nurse home visiting trial (Goldfeld et al. 2017), a unique Australian study that recruited women experiencing adversity during pregnancy and regularly collected self-reported mental health symptoms from pregnancy to 5 years postpartum. The aims of the current study are to (1) describe the patterns of mental health symptoms (depression, anxiety, and stress) from pregnancy to 5 years postpartum and (2) examine how patterns of mental health symptoms differ according to level of antenatal adversity.

\section{Methods}

\section{Design, setting, and participants}

Longitudinal cohort data were drawn from the usual care (control) arm of the "right@ @ome" randomized controlled trial (RCT) of nurse home visiting (Goldfeld et al. 2017). Participants were pregnant women attending antenatal clinics at 10 public maternity hospitals in metropolitan and regional areas of Victoria and Tasmania, Australia, between 30 April 2013 and 29 August 2014. Inclusion criteria were women (1) whose expected due dates were before October 1,$2014 ;(2)$ were less than 37 weeks into their pregnancy at recruitment; (3) had sufficient English proficiency to complete face-to-face interviews; (4) whose home addresses were within the travel boundaries of the study; and (5) who self-reported 2 or more of 10 antenatal adversity risk factors (see Table 1 for details) (Goldfeld et al. 2018a). The study excluded women who (1) were enrolled in an existing Tasmanian nurse home visiting program, (2) did not comprehend the recruitment invitation (e.g., had an intellectual disability such that they were unable to consent to participation or had insufficient English to complete face-to-face interviews), (3) had no mechanism for contact (telephone or email address), or (4) experienced a critical event that excluded their participation (e.g., termination of pregnancy, stillbirth, participant or child death). Those who enrolled in the trial had an overall higher total adversity risk count and were more likely to report poor health, long-term illness, stress anxiety or difficulty coping, and no household income when compared to those who were identified as eligible but chose not to enroll (reasons not provided) (Price et al. 2018).

\section{Procedure}

Eligible women were identified in antenatal clinics and invited into the RCT. Women who chose to enroll provided informed consent and completed a comprehensive baseline home-based interview with a trained researcher, while women were pregnant. The initial RCT consent included follow-up to child age 2 years, at which time women were invited to re-enroll for follow-up to child age 5 years. Maternal mental health was assessed via interview at 11 time-points between May 2013 and Oct 2019, according to child age: at baseline (pregnancy); by phone at 6 weeks 
Table 1 Description of adversity risk factors

\begin{tabular}{|c|c|}
\hline Item & Description \\
\hline Young pregnancy & $\begin{array}{l}\text { Calculated from year of birth and dichotomized into young (" }<23 \text { years") versus not } \\
\text { (" } \geq 23 \text { years") (Kiernan 1997). There were no exclusions for those younger than } \\
18 \text { years }\end{array}$ \\
\hline Not living with another adult & "yes" versus "no" \\
\hline No support in pregnancy & For example, financial, emotional or practical ("yes"/ "no") \\
\hline Poor health & $\begin{array}{l}\text { Single 5-point item ("poor" to "excellent") from the self-reported Short Form-6 (SF6) } \\
\text { (Sanson et al. 2010), dichotomized into poor ("poor/fair/good") versus "very good/ } \\
\text { excellent" health (Mensah and Hobcroft 2008) }\end{array}$ \\
\hline Long-term illness, health problem or disability & $\begin{array}{l}\text { Health problem or disability that limits daily activities ("yes"/ "no"), drawn from the } \\
\text { UK Census } 2001 \text { Census (ONS 2001) }\end{array}$ \\
\hline Currently smokes & "yes" versus "no" \\
\hline Stress, anxiety or difficulty coping & $\begin{array}{l}\text { Matthey two item Generic Mood Question, which has shown good correlation with } \\
\text { longer, validated anxiety measures including the Edinburgh Postnatal Depression } \\
\text { Scale (EPDS) and the Hospital Anxiety Depression Scale (Matthey et al. 2013) } \\
\text { dichotomized into anxious mood versus not }\end{array}$ \\
\hline Low education & $\begin{array}{l}\text { Highest level of school completed, dichotomized into "< Year 12" versus "completed } \\
\text { Year 12" reflecting completion of secondary level education in Australia }\end{array}$ \\
\hline No person in the household currently earning an income & No person in the household currently has paid work/earns an income ("yes"/ "no") \\
\hline Never having had a job before & Participant has never had a job before ("yes"/ "no") \\
\hline
\end{tabular}

postpartum, 6 months, $1.5,2.5$, and 3.5 years; and in participants' homes at $1,2,3,4$, and 5 years.

\section{Measures}

Maternal mental health was assessed at all 11 time-points using the 21-item short form of the Depression Anxiety and Stress Scales (DASS-21) (Henry and Crawford 2005; Lovibond and Lovibond 1995). The DASS-21 is one of the only broad-spectrum, self-report mental health measures and is frequently used in both clinical and public health research (Henry and Crawford 2005). Items are rated on a 4-point scale ("not at all" to "most of the time") assessing symptoms of depression, anxiety, and stress experienced during the past week. Scores are summed to produce a continuous total score (range 0-63) and scores for the three Depression, Anxiety, and Stress subscales (7 items each; range 0-21). Higher scores indicate poorer mental health.

To further explore women's experience of high mental health symptom severity over time, each DASS-21 score was dichotomized to reflect high symptom severity (top $15 \%$ of scores) versus not (below 85\%) according to population reference ranges (UK adults $[\mathrm{N}=1794]$ mean age 41 years, range 18-91 years) (Henry and Crawford 2005). Although the DASS-21 is not a diagnostic tool, the $15 \%$ dichotomy provides important detail of mental health morbidity. A cutpoint of the highest $15 \%$ is in line with common practice for identifying those with a higher clinical burden; a score one standard deviation above the mean according to normative data is equivalent to the 84 th percentile. The proportions of mothers experiencing high symptom severity were explored in addition to considering mental health as a continuum.

Adversity risk was assessed as a total count of the 10 antenatal risk factors surveyed for eligibility (detailed above in inclusion criteria). Antenatal adversity risk was used as a baseline measure of maternal adversity as it is feasible to assess within existing antenatal services, is an important point for initiating support services, and is predictive of later adversity (Price et al. 2018). The adversity risk factor survey was developed by the study team as no existing measures were identified as suitable in the Australian context. Items included in the survey were selected to cover a broad range of known antenatal adversity risk factors, which were feasible to collect in public hospital waiting rooms and acceptable to women. The design, piloting, and data of the adversity risk factor survey are detailed by Price et al. (2017) and Goldfeld et al. (2018a). The survey demonstrated a high degree of co-occurrence between items and identified women experiencing other significant adversities such as housing problems, history of drug and alcohol problems, and family violence (Goldfeld et al. 2018a; Price et al. 2018).

\section{Analysis}

Participant characteristics at baseline (pregnancy) were described and characteristics were compared between those enrolled in the initial RCT usual care arm and those who were retained at the 5-year follow-up; chi-square tests (categorical measures) and t-tests (continuous measures) were used to assess differences arising due to participant attrition. 


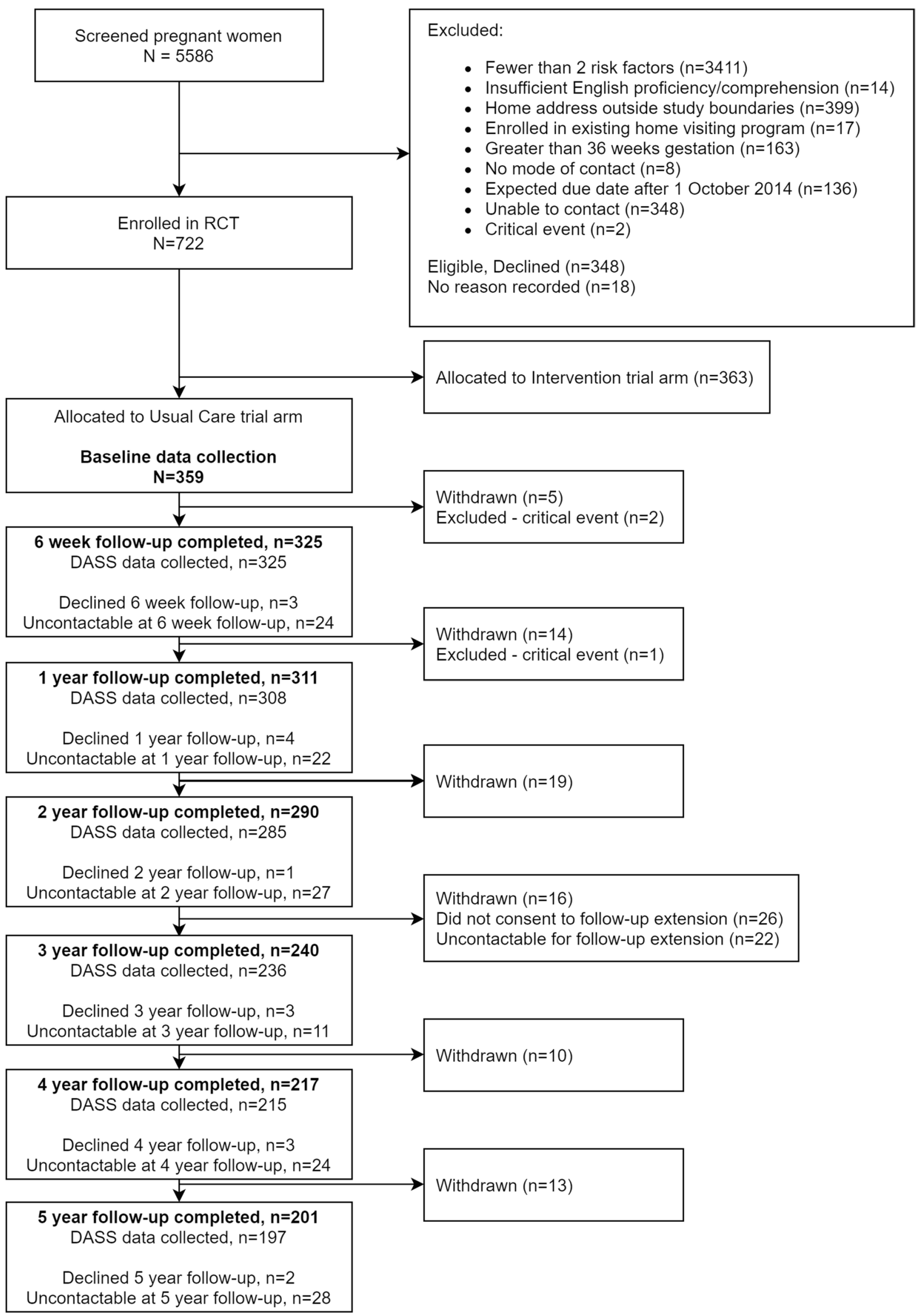


4Fig. 1 Participant consort showing participation rates at baseline, 6-week, and all annual follow-ups

To account for any differences in participant characteristics, arising over time as a result of attrition, data were reweighted at each time-point to be representative of the initial cohort. Weights were derived from the inverse predicted probabilities of participation at each time-point, estimated by logistic regression models which included maternal baseline characteristics of marital status, employment, antenatal adversity risk, age at pregnancy, parity, and mental health as predictors. Weights were applied to all analyses and results are presented using weighted data.

To examine maternal depression, anxiety, and stress symptoms over time, DASS-21 scores at each time-point were summarized for continuous scores (mean and SD) and for dichotomized symptom severity (frequency and percentage). To examine trend associations between higher adversity risk and mental health symptoms, the number of adversity risk factors was categorized into those with 2, 3, 4 , or 5 or more risk factors; a "5 or more" category was used due to small participant numbers with more than 5 risk factors (total adversity range was 2 to 7 in this cohort). DASS-21 total scores were summarized (mean and SD) for each group. Trends in association between adversity risk as a continuous explanatory measure and DASS-21 total score as the continuous outcome were estimated at each time-point using linear regression.

\section{Ethics}

This study was approved by the Human Research Ethics Committee of The Royal Children's Hospital (HREC 32296), Australia.

\section{Results}

The right@ home RCT enrolled 722 pregnant women overall, with 359 allocated to the usual care arm of the trial, which is the focus of this paper. All women $(359,100 \%)$ provided DASS-21 data at baseline (pregnancy) and 343 (96\%) provided DASS-21 data at one or more subsequent time-points (Fig. 1). Women who were uncontactable or declined a particular follow-up were eligible for subsequent follow-up assessments. This allowed women to continue participating even if they missed a follow-up. Women who withdrew or experienced a critical event were excluded from subsequent follow-up.

Table 2 shows the baseline characteristics for the women enrolled in the usual care arm of the RCT in pregnancy and, of these, those who were retained at the 5-year follow-up. Women enrolled and completed the baseline interview at a mean gestation of 28.3 weeks in pregnancy $(\mathrm{SD}=6.8$, range $=11-40$ ); one woman completed the baseline interview 5 days postpartum. Of the 359 enrolled women, 131 (37\%) were first-time mothers and 114 (32\%) reported stress, anxiety or difficulty coping as one of the 10 risk factors included in the screening survey.

Comparison of maternal characteristics between the right@home cohort and published Australian birth cohort data illustrates the high levels of adversity experienced by the right@home cohort: compared to the Longitudinal Study of Australian Children $(\mathrm{N}=5104), 30 \%$ vs. $11 \%$ of households had neither parent in paid employment (Australian Institute of Family Studies 2005); and compared to the Maternal Health Study $(\mathrm{N}=1507), 28 \%$ vs. $5 \%$ were not married or living with a partner, and $67 \%$ vs. $18 \%$ were not in paid employment (Gartland et al. 2010).

Minor differences were observed in the characteristics of those enrolled and those retained at 5 years; those who were retained at 5 years were older at the time of their pregnancy (mean 28.7 years vs. 27.8 years) and less likely to have been unemployed ( $62 \%$ vs. $67 \%$ ). Table 2 also shows baseline demographic and socioeconomic characteristics according to number of antenatal adversity risk factors; greater socioeconomic disadvantage was patterned toward those with higher total antenatal adversity risk.

Across all DASS-21 scales, patterns showed highest mean symptom scores were observed in pregnancy (baseline) and from 4 to 5 years postpartum (Table 3 ). Mean anxiety symptom scores were highest in pregnancy $(\mathrm{m}=3.4, \mathrm{SD}=3.3)$ compared to any other time-point. Mean depression scores were highest at 4 years postpartum $(\mathrm{m}=3.4, \mathrm{SD}=4.0)$, and mean stress and total scores were highest at 5 years $(m=5.9$, $\mathrm{SD}=4.4$ and $\mathrm{m}=12.2, \mathrm{SD}=10.5$, respectively) (Fig. 2). These patterns were also observed in the proportions of women who reported high symptom severity according to dichotomized depression, anxiety, and stress scores over time (Table 3).

Across all levels of antenatal adversity risk, the patterns of mean DASS-21 total symptom scores were similar to that of the whole cohort (Fig. 2); scores were highest in pregnancy and between 3 and 5 years postpartum (Table 4). While the general pattern was comparable across each level of adversity risk, those who had more adversity risk factors in pregnancy had higher mental health symptom scores; a trend association between higher adversity risk count and DASS-21 total score was evident across time (e.g., $\mathrm{p}($ trend $)=0.003$ at baseline; 0.002 at 1.5 years; and 0.013 at 3 years). When considering the overall pattern of scores (Fig. 2), mental health symptoms were particularly pronounced for those with five or more adversity risk factors. These patterns were similar across each of the DASS 
Table 2 Participant characteristics at baseline (pregnancy) by retention status and by adversity risk count

\begin{tabular}{|c|c|c|c|c|c|c|c|}
\hline & \multicolumn{3}{|l|}{ Retention status } & \multicolumn{4}{|c|}{ Adversity risk count } \\
\hline & $\begin{array}{l}\text { Usual care cohort } \\
\mathrm{N}=359\end{array}$ & $\begin{array}{l}\text { Retained at } 5 \text { years } \\
\mathrm{N}=201\end{array}$ & p-value ${ }^{a}$ & $\begin{array}{l}2 \\
n=123\end{array}$ & $\begin{array}{l}3 \\
\mathrm{n}=123\end{array}$ & $\begin{array}{l}4 \\
\mathrm{n}=57\end{array}$ & $\begin{array}{l}5+ \\
\mathrm{n}=56\end{array}$ \\
\hline & Mean (SD) & Mean (SD) & & Mean (SD) & Mean (SD) & Mean (SD) & Mean (SD) \\
\hline \multicolumn{8}{|l|}{ Maternal characteristics } \\
\hline Age at pregnancy, years & $27.8(6.4)$ & $28.7(6.4)$ & 0.002 & $29.5(5.3)$ & $27.5(6.5)$ & $26.1(6.7)$ & $26.7(7.3)$ \\
\hline $\begin{array}{l}\text { Gestation at baseline enrolment, } \\
\text { weeks }\end{array}$ & $28.3(6.8)$ & $28.5(6.8)$ & 0.53 & $28.0(6.9)$ & $28.5(6.5)$ & $27.7(7.2)$ & $28.8(7.0)$ \\
\hline Parity—first child, n(\%) & $131(36.5)$ & $66(32.8)$ & 0.11 & $44(35.8)$ & $49(39.8)$ & $22(38.6)$ & $16(28.6)$ \\
\hline DASS—depression score & $2.9(3.3)$ & $2.9(3.2)$ & 0.75 & $2.3(2.7)$ & $3.0(3.4)$ & $2.6(3.0)$ & $4.3(3.9)$ \\
\hline DASS - anxiety score & $3.4(3.3)$ & $3.3(3.1)$ & 0.50 & $2.9(2.8)$ & $3.4(3.5)$ & $3.6(3.6)$ & $4.5(3.5)$ \\
\hline DASS—stress score & $5.4(4.0)$ & $5.5(4.0)$ & 0.57 & $5.0(3.9)$ & $5.4(4.1)$ & $5.1(4.0)$ & $6.5(4.2)$ \\
\hline DASS—total score & $11.7(9.4)$ & $11.8(9.0)$ & 0.91 & $10.2(8.2)$ & $11.8(9.7)$ & $11.3(9.4)$ & $15.2(10.4)$ \\
\hline Education, $\mathrm{n}(\%)$ & & & 0.95 & & & & \\
\hline $\begin{array}{l}\text { Did not complete high school or any } \\
\text { further education }\end{array}$ & $82(25.3)$ & $46(25.0)$ & & $22(18.8)$ & $21(18.6)$ & $19(40.4)$ & $20(42.6)$ \\
\hline $\begin{array}{l}\text { Completed high school or vocational } \\
\text { training }\end{array}$ & 207 (63.9) & 119 (64.7) & & $73(62.3)$ & $81(71.7)$ & $28(59.6)$ & $25(53.2)$ \\
\hline Completed a university degree & $35(10.8)$ & $19(10.3)$ & & $22(18.8)$ & $11(9.7)$ & $0(0.0)$ & $2(4.3)$ \\
\hline Currently unemployed, n(\%) & $239(66.6)$ & $125(62.2)$ & 0.05 & $63(51.2)$ & $81(65.9)$ & $44(77.2)$ & $51(91.1)$ \\
\hline $\begin{array}{l}\text { Single, not living with partner, sepa- } \\
\text { rated or divorced, } \mathrm{n}(\%)\end{array}$ & 99 (27.6) & $50(24.9)$ & 0.68 & $15(12.2)$ & $31(25.2)$ & $18(31.6)$ & $35(62.5)$ \\
\hline $\begin{array}{l}\text { Family income from benefit or pen- } \\
\text { sion, } \mathrm{n}(\%)\end{array}$ & $150(41.8)$ & $81(40.3)$ & 0.74 & $19(15.5)$ & $46(37.4)$ & $36(63.2)$ & $49(87.5)$ \\
\hline \multicolumn{3}{|c|}{ Area socioeconomic disadvantage (SEIFA quintile), $\mathrm{n}(\%)$} & 0.56 & & & & \\
\hline 1 (greatest disadvantage) & $139(40.2)$ & $80(41.5)$ & & $40(34.2)$ & $49(41.2)$ & $22(39.3)$ & $28(51.9)$ \\
\hline 2 & $30(8.7)$ & $18(9.3)$ & & $12(10.3)$ & $7(5.9)$ & $4(7.1)$ & $7(13.0)$ \\
\hline 3 & $132(38.2)$ & $70(36.3)$ & & 49 (41.9) & $39(32.8)$ & $26(46.4)$ & $18(33.3)$ \\
\hline 4 & $32(9.3)$ & $20(10.4)$ & & $11(9.4)$ & $18(15.1)$ & $3(5.4)$ & $0(0.0)$ \\
\hline 5 (least disadvantage) & $13(3.8)$ & $5(2.6)$ & & $5(4.3)$ & $6(5.0)$ & $1(1.8)$ & $1(1.9)$ \\
\hline \multicolumn{8}{|l|}{ Screening survey risk factors } \\
\hline Total number of risk factors & $3.2(1.2)$ & $3.1(1.2)$ & 0.10 & $2(0.0)$ & $3(0.0)$ & $4(0.0)$ & $5.6(0.7)$ \\
\hline Young age ( $<23$ years), $\mathrm{n}(\%)$ & $101(28.1)$ & $47(23.4)$ & 0.02 & $12(9.8)$ & $39(31.7)$ & $25(43.9)$ & $25(44.6)$ \\
\hline Not living with another adult, n(\%) & $57(15.9)$ & $28(13.9)$ & 0.26 & $9(7.3)$ & $3(7.3)$ & $9(15.8)$ & $30(53.6)$ \\
\hline No support in pregnancy, $\mathrm{n}(\%)$ & $29(8.1)$ & $13(6.5)$ & 0.21 & $2(1.6)$ & $9(7.3)$ & $7(12.3)$ & $11(19.6)$ \\
\hline Poor health, n(\%) & $264(73.5)$ & $153(76.1)$ & 0.21 & $79(64.2)$ & $93(75.6)$ & $41(71.9)$ & $51(91.1)$ \\
\hline Illness or disability, n(\%) & $95(26.5)$ & $57(28.4)$ & 0.36 & $15(12.2)$ & $35(28.5)$ & $23(40.4)$ & $22(39.3)$ \\
\hline Currently smokes, n(\%) & 118 (32.9) & $61(30.4)$ & 0.25 & $17(13.8)$ & $35(28.5)$ & $26(45.6)$ & $40(71.4)$ \\
\hline $\begin{array}{l}\text { Stress, anxiety or difficulty coping, } \\
\mathrm{n}(\%)\end{array}$ & $114(31.8)$ & $70(34.8)$ & 0.16 & $35(28.5)$ & $37(30.1)$ & $18(31.6)$ & $24(42.9)$ \\
\hline Did not complete high school, n(\%) & $226(63.0)$ & $121(60.2)$ & 0.22 & $58(47.2)$ & $76(61.8)$ & $43(75.4)$ & $49(87.5)$ \\
\hline No income, n(\%) & $108(30.1)$ & $55(27.4)$ & 0.21 & $10(8.1)$ & $27(22.0)$ & $26(45.6)$ & $45(80.4)$ \\
\hline Never had a job, n(\%) & $42(11.7)$ & $22(11.0)$ & 0.62 & $9(7.3)$ & $9(7.3)$ & $10(17.5)$ & $14(25.0)$ \\
\hline
\end{tabular}

DASS, Depression Anxiety and Stress Scales; SEIFA, Socioeconomic Index for Areas (Australian Bureau of Statistics Census of Population and Housing 2011)

${ }^{a}$ p-value for chi-square tests (categorical measures) and t-tests (continuous measures) comparing those retained at 5-year follow-up and those lost to follow-up

subscale scores. Higher scores according to adversity risk were most consistent for the Anxiety Subscale, where a trend association was identified at all time-points, while the Stress Subscale showed a trend association at only a single time-point (Supplementary Table 1 and Supplementary Fig. 1). 
Table 3 Descriptive statistics of mental health DASS scale scores over time

\begin{tabular}{|c|c|c|c|c|c|c|c|c|c|c|c|}
\hline & B & 6w & $6 \mathrm{~m}$ & $1 y$ & $1.5 \mathrm{y}$ & $2 \mathrm{y}$ & $2.5 y$ & $3 y$ & $3.5 y$ & $4 y$ & $5 y$ \\
\hline $\mathrm{N}$ & 359 & 325 & 319 & 308 & 297 & 285 & 242 & 236 & 227 & 215 & 197 \\
\hline \multicolumn{12}{|c|}{ Continuous scale scores, mean (SD) } \\
\hline Depression & $2.9(3.3)$ & $2.1(3.1)$ & $1.8(2.5)$ & $2.2(2.7)$ & $1.9(2.8)$ & $2.1(2.9)$ & $2.2(3.1)$ & $3.2(3.5)$ & $2.7(3.2)$ & $3.4(4.0)$ & $3.3(3.7)$ \\
\hline Anxiety & $3.4(3.3)$ & $1.8(2.7)$ & $1.7(2.7)$ & $2.0(2.6)$ & $1.7(2.7)$ & $2.1(2.6)$ & $1.9(2.9)$ & $2.7(3.2)$ & $2.0(2.9)$ & $2.7(3.1)$ & $3.0(3.6)$ \\
\hline Stress & $5.4(4.0)$ & $4.0(4.0)$ & $4.2(4.4)$ & $4.6(3.7)$ & $4.1(4.1)$ & $4.5(3.6)$ & $4.3(3.9)$ & $5.5(4.1)$ & $5.1(4.3)$ & $5.5(4.3)$ & $5.9(4.4)$ \\
\hline Total score & $11.7(9.4)$ & $7.9(8.9)$ & $7.6(8.6)$ & $8.8(7.9)$ & $7.6(8.6)$ & $8.8(7.9)$ & $8.4(8.8)$ & $11.4(9.5)$ & $9.8(9.4)$ & $11.6(10.4)$ & $12.2(10.5)$ \\
\hline \multicolumn{12}{|c|}{ Dichotomized high symptom severity, $\%$} \\
\hline Depression & 15.9 & 8.8 & 10.3 & 9.7 & 9.1 & 10.5 & 12.4 & 24.3 & 15.7 & 23.3 & 24.7 \\
\hline Anxiety & 41.5 & 19.6 & 19.0 & 22.2 & 17.0 & 22.2 & 20.8 & 32.1 & 20.2 & 28.7 & 32.6 \\
\hline Stress & 18.9 & 12.0 & 14.1 & 15.0 & 13.4 & 13.7 & 14.0 & 23.3 & 18.1 & 20.7 & 26.1 \\
\hline Total score & 23.7 & 14.1 & 13.6 & 16.2 & 12.9 & 13.9 & 15.3 & 28.6 & 18.2 & 26.5 & 27.6 \\
\hline
\end{tabular}

$B$, baseline (pregnancy); $6 w, 6$ weeks postpartum; $6 \mathrm{~m}, 6$ months postpartum; $1 y, 1$ year postpartum; $1.5 y, 1.5$ years; $2 y, 2$ years; $2.5 y, 2.5$ years; $3 y, 3$ years; $3.5 y, 3.5$ years; $4 y$, 4 years; $5 y, 5$ years

\section{Discussion}

This study aimed to describe the patterns of mental health symptoms from pregnancy to 5 years postpartum in a cohort of Australian women experiencing adversity. In this unique cohort, we found that mental health symptoms were common across the whole time-period and the pattern of symptoms showed that scores were highest in pregnancy and at 4 to 5 years postpartum. This pattern was consistent for both continuous and dichotomized symptom scores, and across each level of antenatal adversity risk. Within this, there was also a trend in which a higher count of adversity risk factors in pregnancy was associated with higher total mental health symptoms, across most time-points.

Considerable evidence shows that maternal mental health problems are common during pregnancy and the first 12 months postpartum (Shorey et al. 2018; Woody et al. 2017). However, there is a dearth of studies available about mothers' mental health problems beyond this time, and even less available if coupled with the experience of adversity. Our study contributes new evidence to this important gap. Previous research has shown that maternal mental health problems often persist beyond the first year postpartum (Mughal et al. 2018; Putnick et al. 2020) and may actually worsen later (Woolhouse et al. 2015). Our study reinforces these findings, showing a pattern of persistent postpartum mental health symptoms which were highest at 4 to 5 years. This pattern may be explained by an accumulation of persistent and recurring mental health symptoms over time, as well as changing support and family circumstances. While the current study examined adversity only once during pregnancy, families will experience varied patterns of adversity and emerging adversity risks over the postpartum years (Goldfeld et al. 2018b). These changing circumstances, such as relationship transitions, family violence, or loss of income, may contribute to increases in maternal mental health symptoms, as has been identified in previous studies (Woolhouse et al. 2015). The impact of adversity over time is further likely to be cumulative; mothers experiencing adversity during pregnancy are likely to have further experienced sustained or increasing adversity, in which they have been raising a family, throughout these subsequent 5 years.

Our findings further demonstrate how much more prevalent mental health symptoms are for women experiencing adversity than in the general population. In our cohort of women experiencing adversity, 20-30\% of women reported high mental health symptom severity beyond 3 years postpartum. This rate reaches double that of Australian population cohorts; the Longitudinal Study of Australian Children identified $10 \%$ of women as having high depression symptoms at 4 years (Giallo et al. 2014), and the Maternal Health Study reported the highest rate of depression as 15\% at 4 years postpartum (Woolhouse et al. 2015). Moreover, our rates are up to four times greater than a US cohort which identified moderate depression symptoms in $7 \%$ of women at 3 years postpartum (Putnick et al. 2020). Our study further extends on the existing literature by demonstrating similarly high rates of high anxiety and stress symptoms up to 5 years postpartum.

This study has several strengths. We recruited a large cohort of pregnant women specifically for their experience of social and economic adversity. The retention rates achieved $(82 \%$ of participants re-enrolled at 2 years and $56 \%$ retained over the 6-year timespan) are exceptional given the challenges of retaining women experiencing adversity. By comparison with other nurse home visiting trials, by 2-year postpartum follow-up ProKind retained less than 50\% (Sierau et al. 2015), and the French CAPEDP retained $31 \%$ of their original cohort (Foulon et al. 2015). Within our cohort, minimal differences were observed in 
Fig. 2 Mean DASS-21 symptom scores over time and by antenatal adversity risk

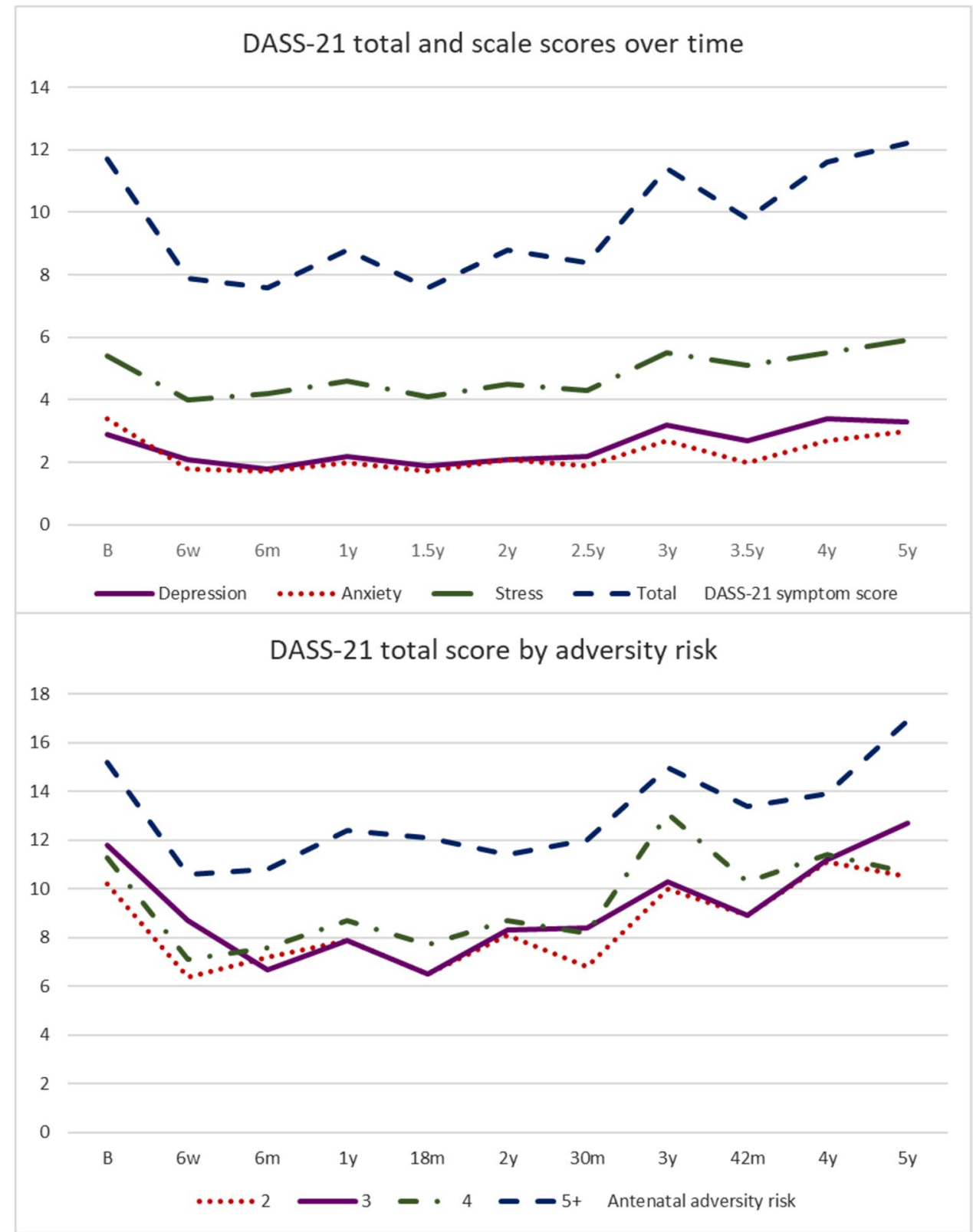

the baseline characteristics between those enrolled and those who were retained at 5 years; in particular, baseline mental health symptoms were similar. Additionally, we used data that were reweighted to be representative of the initial cohort. Therefore, participant attrition is unlikely to account for the changes observed in mental health symptoms over time. Another strength was the repeated assessment of mental health using a broad-spectrum measure to assess symptoms of depression, anxiety, and stress, rather than focusing on a single mental health condition such as depression or anxiety.

There are also several limitations. The DASS-21 is not a diagnostic tool and the cut-points for high mental health symptom severity were study-defined according to normative data. These may not be comparable to rates of mental health conditions reported in existing studies. However, the DASS21 has demonstrated good correlation with the Edinburgh Postnatal Depression Score and other tools which have been validated against diagnostic criteria (Cunningham et al. 2013; Henry and Crawford 2005). With regard to the study cohort, as participants in a larger RCT, these women may not fully represent Australian women experiencing adversity in pregnancy. While those who completed the screening survey and enrolled in the trial reported higher adversity risk than those who declined participation, we do not have data on women who were approached but did not complete the initial screening survey. In addition, our findings may not generalize to women who were excluded from the original trial, 


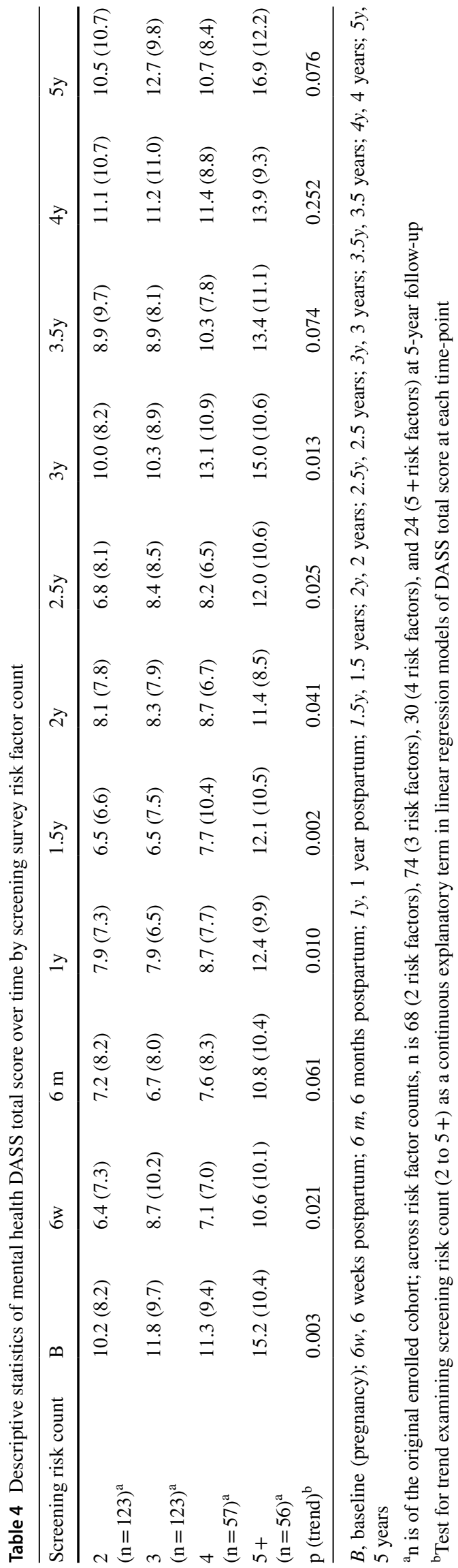

such as those who did not have sufficient English proficiency to complete follow-up assessments, had no mechanism for contact, or cognitive disability. However, given the sampling frame, the findings are generalizable to pregnant women, with sufficient English-speaking proficiency, presenting to public hospitals, who are experiencing adversity.

Understanding the patterns of maternal mental health symptoms, particularly as they impact mothers experiencing adversity and beyond the first year postpartum, provides policymakers with the information needed to craft a more equitable, targeted, and responsive service system during pregnancy, the postpartum period, and early years of raising children. The striking patterns of persistent, high, mental health symptoms powerfully illustrate the ongoing mental health burden. Child and family health services which provide primary healthcare for most mothers are often focused on the immediate postpartum period. Our findings highlight the need for primary health services to sustain family engagement and provide mental health support for mothers throughout the early childhood years. While the current research was not planned with the coronavirus (COVID-19) pandemic in mind, support needs will likely be heightened by global health crises such as the pandemic, which increase the economic and psychosocial stressors for families with young children (Holmes et al. 2020). Delivery of effective and accessible services to mothers, over the early years of their child's life, has potential to improve both women's mental health and have flow-on benefits for the child and family.

Supplementary Information The online version contains supplementary material available at https://doi.org/10.1007/s00737-021-01145-0.

Acknowledgements The "right@home" sustained nurse home visiting trial is a research collaboration between the Australian Research Alliance for Children and Youth (ARACY); the Translational Research and Social Innovation (TReSI) Group at Western Sydney University; and the Centre for Community Child Health $(\mathrm{CCCH})$, which is a department of The Royal Children's Hospital and a research group of Murdoch Children's Research Institute. We thank all families, the researchers, nurses and social care practitioners working on the right@ home trial, the antenatal clinic staff at participating hospitals who helped facilitate the research, and the Expert Reference Group for their guidance in designing the trial.

Funding This work is supported by the state governments of Victoria and Tasmania, the Ian Potter Foundation, Sabemo Trust, Sidney Myer fund, the Vincent Fairfax Family Foundation, and the National Health and Medical Research Council (NHMRC, 1079418). The MCRI administered the research grant for the study and provided infrastructural support to its staff but played no role in the conduct or analysis of the trial. Research at the MCRI is supported by the Victorian Government's Operational Infrastructure Support Program. SG was supported by NHMRC Practitioner Fellowship (1155290). FM was supported by NHMRC Career Development Fellowship (1111160).

Data availability Upon request to the senior author (Sharon Goldfeld: sharon.goldfeld@ @rch.org.au), deidentified participant data, study 
protocols, and statistical analysis plans can be made available after publication to researchers who provide a methodologically sound proposal for use of the data.

\section{Declarations}

Ethics approval This study was approved by the Human Research Ethics Committees of The Royal Children's Hospital (HREC 32296).

Consent to participate Informed consent was obtained from all individual participants included in the study.

Competing interests The authors declare no competing interests.

Disclaimer The funding bodies had no role in relation to the design and conduct of the study; collection, management, analysis, and interpretation of the data; preparation, review, or approval of the manuscript; and decision to submit the manuscript for publication.

\section{References}

Austin M-PV, Hadzi-Pavlovic D, Priest SR, Reilly N, Wilhelm K, Saint K, Parker G (2010) Depressive and anxiety disorders in the postpartum period: how prevalent are they and can we improve their detection? Arch Womens Ment Health 13:395-401

Australian Bureau of Statistics Census of Population and Housing (2011) Socio-Economic Indexes for Areas (SEIFA). Australian Bureau of Statistics, Australia

Australian Institute of Family Studies (2005) The longitudinal study of Australian children annual report 2004. Commonwealth of Australia

Bonevski B et al (2014) Reaching the hard-to-reach: a systematic review of strategies for improving health and medical research with socially disadvantaged groups. BioMed Central Med Res Methodol 14:42. https://doi.org/10.1186/1471-2288-14-42

Cunningham NK, Brown PM, Brooks J, Page AC (2013) The structure of emotional symptoms in the postpartum period: is it unique? J Affect Disord 151:686-694

Dennis CL, Falah-Hassani K, Shiri R (2017) Prevalence of antenatal and postnatal anxiety: systematic review and meta-analysis. $\mathrm{Br}$ J Psychiatry 210:315-323. https://doi.org/10.1192/bjp.bp.116. 187179

Foulon S et al (2015) Predictors of study attrition in a randomized controlled trial evaluating a perinatal home-visiting program with mothers with psychosocial vulnerabilities. PLoS ONE 10:e0142495. https://doi.org/10.1371/journal.pone.0142495

Gartland D, Brown S, Donath S, Perlen S (2010) Women's health in early pregnancy: findings from an Australian nulliparous cohort study. Aust N Z J Obstet Gynaecol 5 doi:https://doi.org/10.1111/j. 1479-828X.2010.01204.x

Giallo R, Cooklin A, Nicholson JM (2014) Risk factors associated with trajectories of mothers' depressive symptoms across the early parenting period: an Australian population-based longitudinal study. Arch Womens Mental Health 17:115-125. https://doi.org/ 10.1007/s00737-014-0411-1

Goldfeld S, D’Abaco E, Bryson H, Mensah F, Price AMH (2018a) Surveying social adversity in pregnancy: the antenatal risk burden experienced by Australian women. J Paediatr Child Health 54:754-760. https://doi.org/10.1111/jpc. 13860

Goldfeld $\mathrm{S}$ et al (2018b) More than a snapshot in time: pathways of disadvantage over childhood. Int J Epidemiol 47:1307-1316. https:// doi.org/10.1093/ije/dyy086
Goldfeld S et al (2017) right@ home: a randomised controlled trial of sustained nurse home visiting from pregnancy to child age 2 years, versus usual care, to improve parent care, parent responsivity and the home learning environment at 2 years. BMJ Open 7:e013307. https://doi.org/10.1136/bmjopen-2016-013307

Hart JT (1971) The inverse care law. The Lancet 297:405-412. https://doi.org/10.1016/S0140-6736(71)92410-X

Henry JD, Crawford JR (2005) The short-form version of the Depression Anxiety Stress Scales (DASS-21): construct validity and normative data in a large non-clinical sample. Br J Clin Psychol 44:227-239. https://doi.org/10.1348/014466505X29657

Holmes EA et al (2020) Multidisciplinary research priorities for the COVID-19 pandemic: a call for action for mental health science. Lancet Psychiatry 7:547-560. https://doi.org/10.1016/S22150366(20)30168-1

Kiernan KE (1997) Becoming a young parent: a longitudinal study of associated factors. Br J Sociol 48:406-428. https://doi.org/ $10.2307 / 591138$

Kingsbury AM, Plotnikova M, Clavarino A, Mamun A, Najman JM (2018) Social adversity in pregnancy and trajectories of women's depressive symptoms: a longitudinal study. Women Birth 31:52-58. https://doi.org/10.1016/j.wombi.2017.06.016

Kingston D, Tough S (2014) Prenatal and postnatal maternal mental health and school-age child development: a systematic review. Maternal and Child Health 18:1728-1741. https://doi.org/10. 1007/s10995-013-1418-3

Ko JY, Haight SC (2020) Addressing perinatal mental health and opportunities for public health. Am J Public Health 110:765-767

Leach LS, Poyser C, Fairweather-Schmidt K (2017) Maternal perinatal anxiety: a review of prevalence and correlates. Clin Psychol 21:4-19. https://doi.org/10.1111/cp.12058

Lovibond SH, Lovibond PF (1995) Manual for the depression anxiety stress scales. (2nd. Ed.) Sydney: Psychology Foundation. http:// www2.psy.unsw.edu.au/dass//

Luca DL, Garlow N, Staatz C, Margiotta C, Zivin K (2019) Societal costs of untreated perinatal mood and anxiety disorders in the United States. Mathematica Policy Research, Cambridge

Matthey S, Valenti B, Souter K, Ross-Hamid C (2013) Research report: comparison of four self-report measures and a generic mood question to screen for anxiety during pregnancy in English-speaking women. J Affect Disord 148:347-351. https://doi.org/10.1016/j. jad.2012.12.022

Mensah F, Hobcroft J (2008) Childhood deprivation, health and development: associations with adult health in the 1958 and 1970 British prospective birth cohort studies. J Epidemiol Community Health 62:599-606

Mughal MK, Giallo R, Arnold P, Benzies K, Kehler H, Bright K, Kingston D (2018) Trajectories of maternal stress and anxiety from pregnancy to three years and child development at 3 years of age: findings from the All Our Families (AOF) pregnancy cohort. J Affect Disord 234:318-326. https://doi.org/10.1016/j.jad.2018. 02.095

ONS (2001) UK Office for National Statistics. Census 2001 key statistics 21: long term illness. http://data.london.gov.uk/dataset/census-2001-key-statistics-21-long-term-illness. Accessed 18 Dec 2015. Office for National Statistics, London

Price A et al (2018) A brief survey to identify pregnant women experiencing increased psychosocial and socioeconomic risk. Women and Birth 32:e351-e358. https://doi.org/10.1016/j.wombi.2018. 08.162

Price A, Bryson H, Mensah F, Kemp L, Bishop L, Goldfeld S (2017) The feasibility and acceptability of a population-level antenatal risk factor survey: cross-sectional pilot study. J Paediatr Child Health 53:572-577. https://doi.org/10.1111/jpc.13510 
PricewaterhouseCoopers, Perinatal Wellbeing Centre, PANDA Australia, Peach Tree Perinatal Wellness (2019) The cost of perinatal depression and anxiety in Australia. North Sydney, NSW

Putnick DL et al (2020) Trajectories of maternal postpartum depressive symptoms. Pediatrics 146:e20200857. https://doi.org/10. 1542/peds.2020-0857

Redshaw M, Henderson J (2016) Who is actually asked about their mental health in pregnancy and the postnatal period? Findings from a national survey. BMC Psychiatry 16:322. https://doi.org/ 10.1186/s12888-016-1029-9

Sanson AV, Hawkins MT, Misson S (2010) The development and validation of australian indices of child development—part II: validity support. Child Indic Res 3:293-312

Shorey S, Chee CYI, Ng ED, Chan YH, Tam WWS, Chong YS (2018) Prevalence and incidence of postpartum depression among healthy mothers: a systematic review and meta-analysis. J Psychiatr Res 104:235-248. https://doi.org/10.1016/j.jpsychires.2018.08.001

Sierau S, Dähne V, Brand T, Kurtz V, von Klitzing K, Jungmann T (2015) Effects of home visitation on maternal competencies, family environment, and child development: a randomized controlled trial. Prev Sci :1-12 https://doi.org/10.1007/s11121-015-0573-8
Stein A et al (2014) Effects of perinatal mental disorders on the fetus and child. Lancet 384:1800-1819. https://doi.org/10.1016/S01406736(14)61277-0

Lancet T (2021) 50 years of the inverse care law. The Lancet 397:797. https://doi.org/10.1016/S0140-6736(21)00505-5

Vigo D, Thornicroft G, Atun R (2016) Estimating the true global burden of mental illness. The Lancet Psychiatry 3:171-178

Woody CA, Ferrari AJ, Siskind DJ, Whiteford HA, Harris MG (2017) A systematic review and meta-regression of the prevalence and incidence of perinatal depression. J Affect Disord 219:86-92. https://doi.org/10.1016/j.jad.2017.05.003

Woolhouse H, Gartland D, Mensah F, Brown SJ (2015) Maternal depression from early pregnancy to 4 years postpartum in a prospective pregnancy cohort study: implications for primary health care. BJOG 122:312-321. https://doi.org/10.1111/1471-0528. 12837

Publisher's note Springer Nature remains neutral with regard to jurisdictional claims in published maps and institutional affiliations. 